\title{
Drag reduction in turbulent flow due to additives: a report on Euromech 52
}

\author{
By F. H. BARK, \\ Department of Mechanics, Royal Institute of Technology, Stockholm
}

E. J. HINCH

Department of Applied Mathematics and Theoretical Physics, University of Cambridge

\section{AND M. T. LANDAHL}

Department of Mechanics, Royal Institute of Technology, Stockholm $\dagger$

(Received 6 December 1974)

Euromech 52, on drag reduction in turbulent flows due to additives, was held from 27-30 August 1974 in Stockholm, Sweden, under the chairmanship of M. T. Landahl, and was intended to bring together specialists in the various aspects of the drag-reduction phenomenon to discuss current experimental and theoretical work, and to identify promising directions for future research. There were forty-seven participants from eleven countries and twenty-two papers were presented, with ample time for discussion. These papers, which are briefly summarized in this report, could be grouped roughly under the headings of $\S \S 2-6$ below.

\section{Introduction}

The phenomenon of drag reduction in a turbulent flow due to certain additives, notably linear polymers of high molecular weight such as polyethylene oxide (Polyox), has been the subject of intensive research during the last fifteen years. The subject is interesting because of potential engineering applications (typically $50 \%$ drag reduction is achieved with only a few parts per million by weight in the case of the most effective polymer additives) and also because an unravelling of the drag-reduction mystery would carry with it an improved understanding of the mechanics of ordinary Newtonian shear-flow turbulence. Several extensive reviews of the subject have appeared in the last few years, emphasizing different aspects of the phenomenon (Lumley 1969; Landahl 1972 $a$; Hoyt 1972a, 1974).

Various models have been proposed to explain the mechanism of drag reduction. Lumley (1969) stresses the change in turbulence scales due to the additive, whereas Landahl (1972a) deals mainly with the effects of the additive

$\dagger$ Also: Department of Aeronautics and Astronautics, Massachusetts Institute of Technology. 
in reducing turbulent energy production. Drag reduction has been observed with many agents. Besides polymers in water and other solvents, fibres (Hoyt 1972b), coarse dust particles in air (Rosetti \& Pfeffer 1972), magnetic fields (Fraim \& Heiser 1967) and flexible walls (Blick et al. 1969) have also been observed to cause reduction in turbulent drag. An important and very difficult aspect of the subject is the study of the rheological properties of polymer solutions and particle suspensions. A recent Euromech Colloquium (no. 49, in Jablonna, Poland, 1974) dealt primarily with this aspect (Hinch \& Ziabicki 1974).

\section{New data on drag reduction}

Many parameters influence drag reduction. In the case of polymer additives the main parameters are concentration and molecular weight. Drag reduction increases rapidly with concentration to a saturation value, and then slowly decreases with further increases in concentration. It depends strongly on the molecular weight, the higher weights (typically $10^{6}$ ) being increasingly effective; on the other hand, mechanical degradation due to scission of the molecules in the turbulent straining field tends to put an upper practical limit on the molecular weight for drag-reduction purposes. Of special importance is the phenomenon of 'onset', namely that the polymer additive becomes effective as a drag reducer first when the wall shear stress exceeds a threshold value, presumably because the randomly coiled molecules first have to be stretched out by the turbulent straining field before drag reduction occurs (Lumley 1972). All such effects tend to make the problem of interpreting drag-reduction measurements for different polymers a very difficult one, particularly since some polymer properties like the distribution of molecular weights are usually not known with precision. For such reasons, published data on drag reduction sometimes appear contradictory.

In the first lecture, P. Gramain (Strasbourg) showed how drag reduction with Polyox was improved by use of solvents that tend to expand the coil. By changing the $\mathrm{pH}$ of the solution he demonstrated that a flexible coiled molecular conformation was superior to a more extended rigid rod-like conformation. Molecular entanglements tended to decrease drag reduction, as did degradation. The thickness of the adsorbed layer of molecules on the solid surface was found to be very small, approximately $200 \AA$, suggesting that the drag reduction cannot be due to wall flexibility caused by the adsorbed polymer layer as suggested by Bryson, Arunachalan \& Fulford (1971). E. H. Dunlop (London) presented pipe-flow drag-reduction measurements for polystyrene in toluene. This polymer, although a considerably less efficient drag reducer than Polyox in water, is interesting because it can be obtained with extremely narrow molecular weight distributions, so that the problems of data interpretation associated with polydispersivity may be largely avoided. Dunlop found increased drag reduction when the temperature was lowered (from $25^{\circ} \mathrm{C}$ to $-17^{\circ} \mathrm{C}$ ). A decrease in temperature produces an increase in the relaxation time of the polymer; these results therefore give support to the time-scale 
hypothesis (Hershey \& Zakin 1965), namely that drag reduction occurs when the typical time scale for the turbulent velocity fluctuations becomes smaller than the relaxation time of the polymer molecule. The question of whether onset is determined by a time or length scale (molecular size/turbulent eddy size) has been one of the crucial problems in the area of drag reduction; the time hypothesis was favoured at this meeting. K. Slanec (Prague) presented dragreduction data for a commercial polyacrylamide called Praestol and had achieved what must be a record testing time for ageing of the solution: he showed that over a period of $4 \frac{1}{2}$ years the effectiveness of this polymer as a drag reducer did not change significantly.

In view of the fact that the additives seem to have their main influence on the structure of the fluctuating field in a region very close to the wall (in the viscous wall layer and in the buffer region), it has been suggested that the polymers may be injected at the wall, thereby reducing the total amount of additive needed. Experiments such as those of Wells \& Spangler (1967) confirm that drag reduction does occur with injection in the wall layer. At Euromech 52, two papers reported on drag reduction by polymer injection. C. Gebel \& H. Reitzer (Strasbourg) measured reduced drag on a flat plate in a pipe with local and distributed injection. They also found that the turbulent mass transport away from the plate was reduced by about the same factor as the drag. J. P. Tullis (Fort Collins) reported measurements of the developing boundary layer in the inlet region of a pipe of very large diameter (12 in.) when the polymer (Polyox) was injected at a distance of, $3 \cdot 5$ diameters from the pipe entrance. The peak drag reduction (about $90 \%$ ) was found to occur at 14 pipe diameters from the inlet and then to level off to the lower value corresponding to homogeneous flow. In a related investigation, J. Vleggaar, P. J. Klijn, F. Kapteijn $\&$ M. Tels (Amsterdam) injected a concentrated polymer solution on the axis of a turbulent pipe flow, forming a long thread in the centre of the pipe. They reported that the drag reduction that occurred was diminished when the flow passed through a constriction causing the thread to disintegrate.

\section{Polymer solutions}

Over the past forty years physical chemists have developed an extensive theory of dilute polymer solutions (for example Ferry 1970, particularly chapter 9). The standard Rouse-Zimm theory is restricted by several linearizations to small distortions of the polymer molecules, and so predicts at typical dragreduction concentrations a small increase in the viscosity which cannot possibly explain the dramatic reduction of turbulent drag. Takserman-Kroser (1963) first showed that in an extensional flow the standard theory must break down when the strain rate exceeds the reciprocal of the longest relaxation time. The breakdown occurs because the equilibrium configuration has a very large distortion leading to a substantial increase in the viscosity, even at a few parts per million for a molecular weight of a million. This molecular behaviour, which is now thought to be typical of almost all linear flows, leads to the time hypothesis for the onset of drag reduction. The Rouse-Zimm model can be adapted 
to large polymer deformations but the mathematics are intractable. E. J. Hinch (Cambridge) presented some new simple models to investigate different aspects of problems of large polymer deformations. Studies (Hinch 1974) of nonlinearities due to the changing hydrodynamic shielding of one part of the chain by another as the polymer deforms revealed a hysteresis: the flow strength required to maintain a large deformation is much weaker than that needed initially to stretch the undeformed random coil, because the large deformation offers a greater length on which the flow can grip. In intermittent turbulence this hysteresis suggests that the time hypothesis for onset should be based on the peak deformation rate and not on an average. A comparison of the polymer backbone strength and the hydrodynamic stretching forces on a greatly deformed polymer led to the prediction that mechanical degradation could easily occur and is almost certain to accompany onset if the degree of polymerization exceeds a fraction of a million. The effects of nonlinear elasticity and hydrodynamies had been studied and were presented by R. I. Tanner (Providence) for a dumb-bell model. An approximation for a peaked configurational distribution yielded simple constitutive equations whose solutions compared well with the exact solutions. Applying the simple equations to an intermittent random velocity gradient demonstrated the hysteresis when the bead friction of the dumb-bell increased with the deformation.

Two papers were concerned with the solubility of Polyox. B. Maijgren (Stockholm) explained why polyethylene oxide is soluble in water while the closely related polymers polymethylene oxide and polytrimethylene oxide (differing only in the number of methyl groups between the oxygen atoms along the backbone) are not soluble. Apparently the separation between the oxygen atoms along the helix of polyethylene oxide matches precisely the separation between oxygen atoms in the ice crystal structure, which is present to some extent in water. E. H. Dunlop (London) showed some measurements of the time development of drag reduction on an immersed rotating disk after some Polyox powder had been added to the tank. A curious overshoot of the drag reduction was tentatively explained in terms of the existence at intermediate times of large clusters which are more efficient drag reducers than isolated molecules.

While a full theoretical description of polymer solutions from a molecular viewpoint is awaited, the solutions can be characterized experimentally. D. F. James (Toronto) described his new rheometer (involving differentially rotating parallel disks with a radial outflow from a central source), which yielded the primary normal-stress difference and hence a fluid relaxation time. His drag-reduction results with the same polymer solutions were rather poorly scaled by the measured relaxation time. The drag-reduction results also showed that the onset point changed with concentration unless the solution viscosity was within $1 \%$ of the solvent viscosity.

J.-M. Piau (Orsay) showed his impressive film of three anomalous flows: Ecoulements de Solutions Diluées de Polymères (distributed by Service du Film Récherche Scientifique, Paris). First a Pitot tube gave a low reading as soon as the strain rate at the stagnation point exceeded a critical value, consistent with the onset time hypothesis. Flow visualization revealed that the fluid 
penetrated the tube when the readings were low. When the strain rate again exceeded a critical value, the flow through a small orifice separating two chambers suddenly dropped, a new flow regime being established with an upstream recirculating eddy (or vortex) which was not always steady. Finally the flow past a cylinder showed no Kármán vortex street and the forward stagnation point left the surface of the cylinder and moved upstream into the fluid. A. Lewkowicz (Liverpool) confirmed that Pitot tubes were not useful in polymer solutions. He had found that the tubes could not be recalibrated because the pressure reading in a particular polymer solution depended on the velocity shear as well as on the velocity.

Another flow phenomenon which, like drag reduction, is of potential practical importance is the inhibition of eavitation by the addition of polymers. J. H. J. van der Meulen (Wageningen) described some cavitation experiments in which polymer was injected from the nose of a bluff body; he found inhibition of cavitation for a strainless-steel model but not for a Teflon model. It was suggested that the difference might be due either to the cavitation with the Teflon being dominated by surface nucleation, or to the surface chemistry having some effect on the evenness of spread of the injected polymer.

\section{Additional fluid flow systems with drag reduction}

A calculation of the skin friction for turbulent flows of conducting liquids in channels and pipes with a strong aligned magnetic field was reported by $\mathbf{E}$. R. Lindgren \& R. Elkins (Stockholm and Gainsville). The equations of motion were used to determine the relevant combination of similarity parameters. The results were found to agree satisfactorily with the measurements by Fraim \& Heiser (1967). An investigation of the same flow situation from a somewhat different point of view was presented by H. Gustavsson (Stockholm). In this case, the turbulence model rests on the assumption that the turbulent bursts, which are known to be responsible for the major part of the momentum transport, are the results of a hydrodynamic instability of the Kelvin-Helmholtz type. The calculations by Gustavsson assumed a perfectly conducting wall and took account of the finite curvature of the quasi-steady velocity profile just before bursting. The results showed that the instability waves are strongly stabilized by the magnetic field and that, according to the turbulence model used, drag reduction should occur owing to inhibition of bursting.

A fluid-additive system of great interest from the theoretical point of view is dust in air, because of the relative confidence with which the bulk rheological properties may be modelled (in contrast to the situation with polymer solutions). In such dilute suspensions, drag reductions of up to $75 \%$ have been reported (Rosetti \& Pfeffer 1972) with particle sizes in the range $0-70 \mu \mathrm{m}$ and mass loading ratios of order unity. R. Pfeffer (New York) gave an account of the pioneering work carried out within his group on drag reduction with glass beads suspended in air. He pointed out the experimental difficulties associated with sedimentation of the particles in a horizontal test section, and he demonstrated that gas-solid suspensions show substantial similarities to polymer 
solutions in their drag-reduction properties. In particular, drag reduction is accompanied by a thickening of the sublayer without a change in the Kármán constant in the logarithmic velocity profile. Also the turbulent intensities normalized with the wall friction velocity are increased above their Newtonian values. The adaptation of the turbulence might be qualitatively similar for dusty gases and polymer solutions, but the rheologies are essentially different, dissipation being increased on the one hand by the slip of inertial dust relative to the fluid and on the other by the deformation of polymer molecules.

\section{Flow structure}

The central and perhaps the most difficult problem in the drag-reduction field is to describe and understand the effects of additives on the turbulence structure. This requires knowledge of the mechanics of ordinary Newtonian shear-flow turbulence that is not yet available, but towards which some progress has been made in recent years. Visual observations in a thick turbulent boundary layer (Kline et al. 1967; Corino \& Brodkey 1969; Kim, Kline \& Reynolds 1971) have revealed that localized 'bursting' is responsible for the creation of most of the turbulent stresses. Such results have stimulated the development of selective sampling techniques to study the properties of bursts. Blackwelder \& Kaplan (1972) showed by this technique that the sublayer velocity profile is highly inflexional during a burst; the measurements of Morrison, Bullock \& Kronauer (1971) indicate that the large-scale velocity fluctuations have a highly swept wavelike structure. For flows with dragreducing additives, laser-Doppler measurements by Rudd (1972) confirmed earlier results that the wall-layer thickness increases with drag reduction and showed that the fluctuation intensities increase, so that drag reduction is not associated with quenching of the turbulence. Donohue, Tiederman \& Reischmann (1972) found that polymer additives increased the lateral spacing of the low-speed streaks in the wall layer preceding bursting, and that the number of bursts per unit area decreased as did the intensity of the bursts.

A session of the Colloquium was devoted to the structure of shear-flow turbulence in Newtonian and drag-reducing flows. Measurements of the sublayer structure in a turbulent shear flow of pure water and a dilute polymer solution were reported by A. Gyr (Zurich). Two methods were used to detect the structure of the fluctuating velocity field near the wall. The first was an examination of the pattern formed by fine-grained sand at the bottom of a suddenly tilted trough of water. The boundary layer was artificially tripped by a transverse wire. The second experiment involved visual observation of hydrogen bubbles in a turbulent channel flow. From both these experiments Gyr concluded that for pure water the fluctuating velocity field consists to a large degree of highly swept waves as suggested by Morrison et al. (1971). For the drag-reduction case, however, Gyr found that the wave pattern becomes almost two-dimensional, i.e. the wave fronts were most of the time nearly perpendicular to the mean flow direction.

Extensive measurements of the small-scale structure of turbulence in a 
boundary layer with zero pressure gradient were reported by F. N. Frenkiel \& P. Klebanoff (Bethesda). These experiments were performed in order to obtain information about the statistical properties of small-scale motions. Such motions occur intermittently almost everywhere in the boundary layer. Moments of up to fourteenth order of the square of the time derivative of the streamwise velocity fluctuation were measured by using hot-wire anemometry and digital data processing on a high-speed computer. The probability distribution for a non-negative, but otherwise arbitrary, small-scale fluctuating quantity in high Reynolds number turbulence has been shown theoretically under certain assumptions to be lognormal by Gurvich \& Yaglom (1967). This probability distribution was postulated earlier by Kolmogorov (1962) in an attempt to describe the 'spotty' dissipative motions in isotropic turbulence. Gurvich \& Yaglom also found that moments of arbitrary order should be given by a simple formula involving the order of the moment, the mean value and the variance of the logarithm of the fluctuating quantity under consideration. Frenkiel showed that the measurements were consistent with these theoretical predictions. Some disagreements could be attributed to inaccuracies in the measured tails of the probability distributions; it may be, however, that the lognormal distribution is inapplicable (Kraichnan 1974; Mandelbrot 1974).

E. R. Lindgren \& R. R. Johnson (Stockholm and Gainsville) reported some measurements of similarity properties of low Reynolds number turbulence and discussed the reproducibility of averaged quantities in such flows. Also a time sequence of three-dimensional maps of the three components of velocity in a turbulent pipe flow was obtained by a novel technique using a stereoscopic ciné film. The measurements indicated that the fluctuating velocity field in the core region results from the breakdown of small-scale, highly rotational, motions near the wall (which might be closely related to the bursting process). During breakdown a strong growth of scales of motion takes place ("anticascading'), exciting large-scale fluctuations in the core region. 'These results are akin to those of Corino \& Brodkey (1969) and Kim et al. (1971). The largescale eddies were found to be not self-sustaining but decaying as they travelled downstream. New large eddies were created in a random manner by the breakdown mechanism.

\section{Flow models}

Most of the flow models employed so far in efforts to explain drag reduction have been of a qualitative and phenomenological nature; the elastic sublayer model of Virk (1971) is an example. Dynamic models for Newtonian shear-flow turbulence have been constructed by Landahl $(1967,1972 b)$, the latter being based on a subdivision of the fluctuation field into a small-scale 'active' part producing the turbulent stresses and a large-scale 'passive' part having partially the character of decaying waves. Extensions to drag-reducing flows have been put forward by Landahl (1972a) and Landahl \& Bark (1974).

At the Colloquium two papers on flow models were given. A phenomenological model for momentum and heat transfer in flows with drag reduction had been 
studied by Y. Dimant \& M. Poreh (Haifa). M. Poreh pointed out that the usual approximations of constant momentum flux and heat flux in the wall region cannot be used for low Reynolds number turbulence with large drag reduction. Using a modified version of the mixing-length model proposed by van Driest (1956), Poreh showed that very good agreement with available data could be obtained. Also the effects on fluid properties of heating and of non-uniform degradation in planes perpendicular to the mean flow were shown to be of importance when interpreting experimental data.

F. H. Bark (Stockholm) presented some model calculations of one- and two-dimensional wavenumber-frequency spectra of the streamwise velocity fluctuation in the wall layer of a turbulent shear flow of a Newtonian fluid. The large-scale fluctuations dominating the long-time averages were assumed to be excited by small-scale 'bursting' motions, following a statistical model similar to that of Kovasznay (1973). The results showed good qualitative agreement for all three one-dimensional spectra and for the frequency-streamwise wavenumber spectrum when compared with the measurements by Morrison et al. (1971). Also the convection velocity and the decay rate were reasonably well predicted in the manner proposed by Landahl (1967).

\section{Conclusions}

In the general discussion at the close of the Colloquium, C. Elata expressed the opinion that research on drag reduction should aim primarily at 'firstorder' phenomena such as the very large reduction in friction drag achieved with exceedingly small amounts of polymer. 'Second-order' phenomena such as effects of degradation of polymers, although of great practical importance, are not relevant to attempts to arrive at a mechanistic explanation of drag reduction. This opinion seemed to be shared by the other participants, who considered at length the most desirable directions for research in the two problem areas central to the drag-reduction phenomenon, namely, the rheology of suspensions and turbulence structure.

The practical aspects of drag reduction were not altogether forgotten at Euromech 52. During an afternoon boat excursion in the Stockholm archipelago R. 'Tex' Lindgren conducted a drag-reduction experiment by pouring a liquid labelled Polyox into the sea at the bow of the steamer. The participants noticed a definite increase in the speed of the ship. This was also confirmed by the ship's captain!

\section{REFERENCES}

(An asterisk by a name indicates a lecture given at the Colloquium)

BARK, F. H. 1974 On the wave structure of turbulent boundary layers with application to drag reduction. Ph.D. thesis, Department of Mechanics, Royal Institute of Technology, Stockholm, Sweden.

BARk, F. H.* The wave structure of turbulent boundary layers with application to drag reduction.

BLACKWELder, R. F. \& KAPLAN, R. E. 1972 The intermittent structure of the wall region of the turbulent boundary layer. University of Southern California Rep. USCAE 1-22. 
Blick, E. F., Walters, R. R., Smith, R. \& ChU, H. 1969 Compliant coating skin friction experiments. A.I.A.A. Paper, no. 69-165.

Bryson, A. W., Arunachalan, V. R. \& Fulford, G. D. 1971 A traeer dispersion study of the drag-reducing effect in a turbulent pipe flow. J. Fluid Mech. 47, 209.

Corino, E. R. \& Brodkey, R.S. 1969 A visual investigation of the wall region in turbulent flow. J. Fluid Mech. 37, 1.

Dimant, Y. \& PoReh, M. * Phenomenological models of momentum and heat transfer in flows with drag reduction.

Donohue, G. L., Tiederman, W. G. \& Reischmann, M. M. 1972 Flow visualization of the near-wall region in a drag-reducing channel flow. J. Fluid Mech. 56, 559.

Driest, M. T. van 1956 On turbulent flow near a wall. J. Aero. Sci. 23, 1007.

DUNLOP, E. H.* Drag reduction with polystyrene in toluene.

Dunlor, E. H.* The role of molecular aggregates in drag reduction.

Ferry, J. D. 1970 Viscoelastic Properties of Polymers, 2nd ed. Wiley.

Fraim, F. W. \& HeIser, W. H. 1967 The effect of a strong longitudinal magnetic field on the flow of mercury in a circular pipe. J. Fluid Mech. 33, 397.

Frenkiet, F. N. \& KLebanofF, P. S.* Small-scale structure of turbulence in the boundary layer.

Gebet, C. \& Reitzer, H.* Injection parietale de fluides non-Newtoniens dans la couche limite turbulente: reduction de frottement.

Gramain, P.* Polymer solutions and their effectiveness in drag reduction.

Gurvich, A. S. \& YaGlom, A. M. 1967 Breakdown of eddies and probability distributions for small-scale turbulence. Phys. Fluids, 10, 559.

Gustavsson, H.* Effect of a magnetic field on stability of small-scale motion in a turbulent shear flow.

GYR, A.* Studies on the interaction of dilute polymer solutions with movable sand beds in the sublayer region.

HersheY, H. C. \& ZaKIN, J. L. 1965 Study of the turbulent drag reduction of solutions of high polymers. 58th Meeting A.I.Ch.E., Philadelphia, preprint 21B.

HiNCH, E. J. 1974 Mechanical models of dilute polymer solutions for strong flows with large polymer deformations. C.N.R.S. Coll. Polymères \& Lubrification, Brest, France.

HiNCH, E. J.* Rheological models for polymer solutions.

HINCH, E. J. \& ZIABICKT, A. 1974 The mechanics of fluid suspensions and polymer solutions: a report on Euromech 49. J. Fluid Mech. 66, 1.

Hoyt, J.W. 1972a The effect of additives on fluid friction. J. Basic Engng, Trans. A.S.M.E. D94, 258.

Hoy, J. W. $1972 b$ Turbulent flow of drag reducing suspensions. Naval Undersea Centre Rep. NUC TP 299.

Hoyt, J. W. 1974 Recent progress in polymer drag reduction. C.N.R.S. Coll. Polymères de Lubrification, Brest, France.

J AMES, D. F. \& Truony, Q. S.* Measurements of rheological properties and drag reduction of Polyox solutions.

KrM, H. T., KLINe, S. J. \& Reynolds, W. C. 1971 The production of turbulence near a smooth wall in a turbulent boundary layer. J. Fluid Mech. 50, 133.

Kunne, S. J., Reynomins, W. C., Schraub, F. A. \& Runstadier, P. W. 1967 The strueture of turbulent boundary layers. J. Fluid Mesh. 30, 741.

KolMogorov, A.M. 1962 A refinement of previous hypotheses concerning the local structure of turbulence in a viscous incompressible fluid at high Reynolds numbers. J. Fluid Mech. 13, 82.

Kovasznay, L. S. G. 1973 Turbulent shear flows. Symposia Matematica, 9, 507.

Kraichnan, R. H. 1974 On Kolmogorov's inertial-range theories. J. Fluid Mech. 62, 305.

LANDAHL, M. T. 1967 A wave guide model for turbulent shear flow. J. Fluid Mech. 29, 441. 
Landahl, M. T. $1972 a$ Drag reduction by polymer addition. Proc. 13th IUTAM Congr. (ed. E. Becher \& G. R. Mikhailov). Springer.

LaNDAHL, M. T. $1972 b$ Wave mechanies of breakdown. J. Fluid Mech. 56, 775.

LANDAHL, M. T. \& BARK, F. H. 1974 Application of a two scalo boundary layer turbulence model to drag reduction. C.N.R.S. Coll. Polymères \& Lubrification, Brest, France.

LEwkowicz, A.* The Pitot-tube errors in shear flows of drag-reducing polymer solutions.

Lindgren, E. R. \& Elkins, R.* Drag reduction in conducting fluids with a longitudinal magnetic field.

LINDGREN, E. R. \& Johnson, R. R.* Detailed investigation of turbulence structure in pipe flows.

Lumley, J. L. 1969 Drag reduction by additives. Ann. Rev. Fluid Mech. 1, 367.

LumLeY, J. L. 1972 On the solution of equations describing small scale deformation. Symposia Mathematica, 9, 315.

MaIJGRen, B.* Molecular aspects of the drag-reduction phenomenon.

Mandelbrot, B. B. 1974 Intermittent turbulence in self-similar cascades: divergence of high moments and dimension of the carrier. J. Fluid Mech. 62, 331.

MEULEN, J. H. J. VAN DER* Frietion reduction and degradation of polymer additives and the influence on cavitation.

Morrison, W. R. B., Bullock, K. J. \& Kronauer, R. E. 1971 Experimental evidence of waves in the sublayer. J. Fluid Mech. 47, 639.

Pfeffer, R.* Characteristics of dilute gas-solids suspension in drag-reducing flow.

PIAU, J. M.* Diluted polymer solutions flow.

Rosetti, S. J. \& Pfeffer, R. 1972 Drag reduction in dilute flowing gas-solid suspensions. A.I.Ch.E. J. 18, 31 .

RUDD, M. J. 1972 Velocity measurements made with a laser dopplermeter on the turbulent pipe flow of a dilute polymer solution. J. Fluid Mech. 51, 673 .

Stanec, K.* Verringenung der hydraulischen Verluste und der Abbau der makromolekularen Additiven.

TAkserman-Krozer, R. 1963 Behaviour of polymer solutions in the velocity field with parallel gradient. III. Molecular orientation in dilute solutions containing flexible chain macromolecules. J. Polymer Sci. A1, 2477.

TANNer, R. I.* Stresses in dilute solutions of nonlinear bead-spring molecules.

TuluIs, J. P.* Drag reduction and velocity profiles development with polymer injection.

VIRK, P.S. 1971 An elastic sublayer model for drag reduction by dilute solutions of linear macromolecules. $J$. Fluid Mech. 45, 417.

VleggaAR, J., KLIJN, P. J., KapteiJn, F. \& Tels, M.* Flow studies on homogeneous and heterogeneous drag reduction.

Wells, C. S. \& SPangler, J. G. 1967 Injection of a drag-reducing fluid into turbulent pipe flow of a Newtonian fluid. Phys. Fluids, 10, 1890. 